\title{
Report:
}

\section{Diagnosis and treatment of allergic diseases in Zhejiang Province: a cross-sectional survey ${ }^{*}$}

\author{
Hui-ying $\mathrm{WANG}^{\dagger \S 1}$, Yi-hong SHEN ${ }^{\S 2}$, Xu-yan $\mathrm{YANG}^{3}$, Lan-fang TANG ${ }^{4}$, Jian-ying ZHOU ${ }^{\dagger \ddagger 2}$ \\ on behalf of the Zhejiang Provincial Academy of Allergy \\ $\left({ }^{1}\right.$ Department of Allergy, the Second Affiliated Hospital, School of Medicine, Zhejiang University, Hangzhou 310009, China) \\ ('Department of Respirology, the First Affiliated Hospital, School of Medicine, Zhejiang University, Hangzhou 310003, China) \\ $\left({ }^{3}\right.$ Department of Rheumatology and Clinical Immunology, the Second Affiliated Hospital, School of Medicine, \\ Zhejiang University, Hangzhou 310009, China) \\ $\left({ }^{4}\right.$ Department of Respirology, Children's Hospital, School of Medicine, Zhejiang University, Hangzhou 310006, China) \\ †E-mail: marywang@zju.edu.cn; zjyhz@zju.edu.cn \\ Received Oct. 23, 2014; Revision accepted Mar. 24, 2015; Crosschecked June 10, 2015
}

\begin{abstract}
Background: The specialty of allergy developed quickly in western countries because of the rapid increase of allergic diseases, whereas it developed relatively slowly in China. The prevalence of allergen sensitization and allergic diseases in Zhejiang Province of China is high and improving the medical services for these diseases is critically needed. Objective: To investigate the working status of the diagnosis and treatment of allergic diseases, including doctor resources, diagnostic methods, and allergen-specific immunotherapy in patients of Zhejiang Province, and to provide instructions for the strategic development of subspecialties of allergic diseases. Methods: First we defined the doctors who treat allergic diseases, and designed a comprehensive questionnaire to collect personal and hospital information for these doctors. The questionnaires were distributed to hospitals with different ranks and from different areas in the province. The general condition of doctor's resources, carryout of diagnostic methods, and allergen-specific immunotherapy were described and variations in the different specialties, hospitals, and areas were further analyzed. Results: Doctors in their thirties with bachelor's degrees were the mainstream for diagnosing and treating allergic diseases. The main specialties of the doctor resources were the specialties of Ear, Nose and Throat (ENT), Respirology, Pediatrics, and Dermatology. The Pediatrics specialty had a more reasonable infrastructure of doctor resources with more young doctors working in this subspecialty. The development of allergy subspecialty varied within hospitals at different levels or from different areas. The carryout of the skin prick test (SPT), serum specific lgE (sslgE), and subcutaneous immunotherapy (SCIT) was best performed in provincial hospitals, while sublingual immunotherapy (SLIT) was prescribed most commonly in municipal hospitals. The performance of SPT and SslgE in Hangzhou, Jiaxing, and Wenzhou areas was much better than that in other places. The performance of SCIT and SLIT was best in Wenzhou. Conclusions: Our survey revealed a very initial and unbalanced development for the allergy subspecialty in Zhejiang Province. Doctor resources for allergic diseases were mainly from the specialties of ENT, Respirology, and Pediatrics, and the performance of diagnosis and treatment was mainly focused on provincial and municipal hospitals. Continuous education of allergies could be extended to primary healthcare centers and more efforts should be directed to those areas with poor medical resources.
\end{abstract}

Key words: Allergy, Diagnosis, Treatment, Allergen-specific immunotherapy doi: $10.1631 /$ jzus.B1400284

Document code: A

CLC number: R593.1

\footnotetext{
¿ Corresponding author

$\S$ The two authors contributed equally to this work

* Project supported by the National Natural Science Foundation of China (No. 30600266), the Zhejiang Provincial Science and Technology Project (No. 2011C37073), the Zhejiang Provincial Natural Science Foundation (No. LQ12H16012), the National Key Clinical Project of Allergy of China, and the National Key Clinical Specialist Construction Programs of China

\# Electronic supplementary materials: The online version of this article (http://dx.doi.org/10.1631/jzus.B1400284) contains supplementary materials, which are available to authorized users

(1D ORCID: Hui-ying WANG, http://orcid.org/0000-0001-8630-2592; Jian-ying ZHOU, http://orcid.org/0000-0002-3150-5031

(C) Zhejiang University and Springer-Verlag Berlin Heidelberg 2015
} 


\section{Introduction}

The prevalence of allergic diseases has increased rapidly worldwide and has caused a significant social economic burden for healthcare systems during the past three decades (Asher et al., 2006; Zuberbier et al., 2014), which requires the growing needs for medical services related to allergy and clinical immunology. The clinical subspecialty of allergic studies has developed quickly with the significant increases in international organizations and domestic associations for allergic studies such as the World Allergy Organization (WAO), Global Allergy and Asthma European Network (GA $\left.{ }^{2} \mathrm{LEN}\right)$, European Academy of Allergy and Clinical Immunology (EAACI), and American Academy of Allergy, Asthma \& Immunology (AAAAI) (Hurwitz, 1953; Warner et al., 2006; Bergmann, 2014). These academic organizations for allergic studies have contributed greatly to the development of the subspecialty through professional physician-training and guideline-releases (Cox et al., 2011; Bousquet et al., 2012). As a result, laboratory exams of allergen diagnosis and allergen-specific immunotherapy have been carried out extensively through clinical practices, which play important roles in treating allergic diseases (Krouse and Mabry, 2003; Burks et al., 2013; Bergmann, 2014; Fornadley, 2014). It should be noted that an allergy/immunology physician workforce is growing steadily with increasing diversity and occupational satisfaction (Marshall, 2007).

However, the clinical subspecialty of allergies in China has developed relatively slow. Although the first recognition of allergies was introduced by Dr. Qing-song ZHANG in 1953, most of the doctors in China are still unfamiliar with this subspecialty (Ye, 2001). The National Academy of Allergy is small in size and has a weak impact, and it has only released a few standard regulations or guidelines that could be used for instructing clinical work. As a matter of fact, independent Departments of Allergy are scattered throughout even provincial or teaching hospitals, and allergists/immunologists that have accepted professional training are extremely rare (Yin, 2014). Patients with allergic diseases usually have to go to different departments based on the organ systems affected. For example, patients of allergic rhinitis go to Ear, Nose and Throat (ENT) Departments, patients of asthma to Respiratory Departments, and patients of atopic dermatitis to Dermatology Departments, respectively. Thus, treatments usually focus on medications for symptom relief, but the underlying immunologic mechanisms are often ignored. This underdeveloped system restricts the clinical applications of allergen-specific immunotherapy.

In addition, the development of allergy subspecialties is also geographically unbalanced in China, being relatively strong in the North (Wen, 2013). Totally less than 20 provincial academies of allergic studies have been set up, and that is primarily in northern China. Zhejiang Province is an eastern coastal province, close to Shanghai, with well-developed economics and a large population (Zhejiang Provincial Bureau of Statistics, 2014), as well as a high prevalence of allergic diseases and allergen sensitizations (Li et al., 2009). However, medical service for accurate diagnosis and efficient treatments for allergic diseases is still at a low level and is in need of significant improvement. So after the foundation of the Zhejiang Provincial Academy of Allergy, we immediately conducted a survey on the status of the diagnosis and treatment of allergic diseases, including doctor resources, performance of diagnostic methods and allergen-specific immunotherapy, with the desire to collect essential information for providing instructions for the future strategic development of subspecialties of allergic diseases.

\section{Materials and methods}

To accomplish this survey, the first step was to define the doctors who treat allergic diseases, considering the scarcity of allergists/immunologists and the large population of doctors who could diagnose patients with allergic diseases and prescribe medications to relieve their symptoms. We defined the doctors who treat allergic diseases as the doctors who could interpret the results of allergen measurement and/or perform allergen-specific immunotherapy. A questionnaire was designed to collect demographics for these doctors including personal information such as sex, age, academic degrees, work-time, professional rank, the specialties and hospitals where they were working, and which allergic diseases they treat. Meanwhile, hospital information such as the rank and 
the performance of diagnostic methods and allergenspecific immunotherapy was also required to be completed as part of the questionnaire. According to the real conditions in China, diagnostic methods were classified into skin prick test (SPT) and serum specific IgE (ssIgE). And the carryout of ImmunoCAP, belonging to the ssIgE measurement, was also investigated. Allergen-specific immunotherapy was classified as sublingual immunotherapy (SLIT) and subcutaneous immunotherapy (SCIT).

These questionnaires were distributed to hospitals ranked as provincial hospitals, municipal hospitals, county hospitals, and district hospitals according to their administration management, which usually correlated to the academic level and hospital size. Rural country healthcare units were not included. As Zhejiang Province is divided into nine geographic areas, i.e. Hangzhou, Huzhou, Jiaxing, Jinhua, Ningbo, Quzhou, Shaoxing, Taizhou, and Wenzhou, nine professors from each area were chosen to take responsibility for distributing the questionnaires to the doctors who treated allergic diseases, as we defined in local hospitals of corresponding areas, and to collect them after completion.

Data of age was expressed as mean value, while other information was expressed as a percentage. The general conditions of the doctor resources and medical services of diagnostic methods and allergen-specific immunotherapy were described and the variation in different specialties, hospitals, and areas was further analyzed.

\section{Results}

\subsection{General characteristics and distribution of doctors treating allergic diseases}

A total of 357 doctors from 69 hospitals completed the questionnaire survey, consisting of 204 men and 153 women. The average age of the doctors was 38.93 years, ranging from 25 to 64 years. Doctors in their thirties were the mainstream in diagnosing and treating allergic diseases, followed by doctors in their forties and fifties. The ratio of young doctors under 30 years old was relatively low as shown in Fig. 1a. More than $65 \%$ of the doctors had bachelor's degrees, and the proportion of doctors with master's and doctorate's degrees was more than $30 \%$ (Fig. 1b). Mu- nicipal hospitals included the highest proportion of doctor resources, followed by county and provincial hospitals (Fig. 1c). It should be noted that there were also doctors in district hospitals treating allergic diseases. The main resources of doctors were the Departments of ENT, Respirology, Pediatrics and Dermatology (Fig. 1d). Only one independent Allergy Department was set up in a provincial hospital affiliated with the Zhejiang University School of Medicine, Hangzhou, China. Departments of Internal Medicine in some municipal or county hospitals were also one resource of doctors treating allergic diseases. And the data of area distribution showed the highest percentage of hospitals and doctors treating allergic diseases in Hangzhou, followed by Ningbo and Taizhou areas, while the Jiaxing area had the lowest number of hospitals and doctors (Fig. 1e). The percentage of hospitals in the Quzhou and Wenzhou areas was more than $10 \%$; however, the percentage of doctors in these two areas was much lower. By contrast, in Jinhua and Shaoxing areas, the percentage of doctors was much higher than that of associated hospitals. The common allergic diseases were allergic rhinitis, allergic asthma, atopic dermatitis, and allergic pneumonia. Only doctors from the Department of Allergy provided comprehensive medical service for allergic diseases. Doctors from the specialties of Pediatrics and Internal Medicine usually treated allergic rhinitis and asthma, while doctors from the specialties of ENT, Respirology, and Dermatology only treated allergic rhinitis, asthma, and atopic dermatitis, respectively.

\subsection{Infrastructure of doctor resources in the spe- cialties of Respirology, ENT, and Pediatrics}

We further examined the infrastructure of doctor resources in three main specialties. The percentages of male doctors were higher than that of female doctors in the specialties of Respirology and ENT, while women were more prevalent than men in the specialty of Pediatrics as shown in Fig. 2a. Age distribution of doctors in the specialties of Respirology and ENT was similar to that of the general population with the peak number of doctors being in their thirties, and a lower number for those who were less than their thirties and those in their forties. In the ENT specialty, the percentage of doctors in their thirties and less than 30 years old were dramatically high (52\%) and low $(8 \%)$, respectively. However, in the Pediatrics specialty, the 

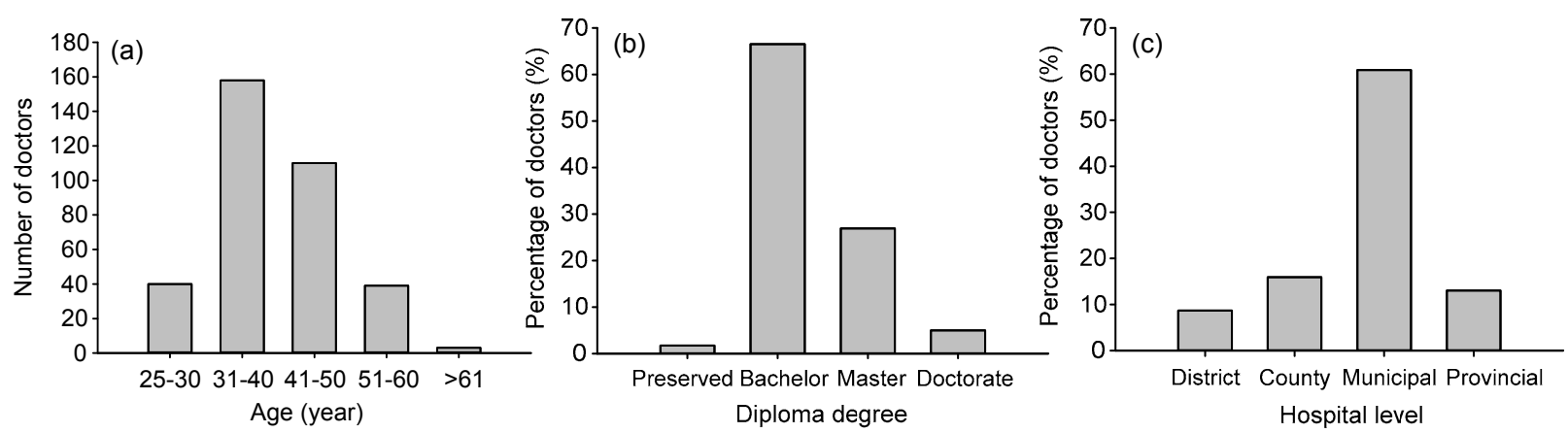

(d)

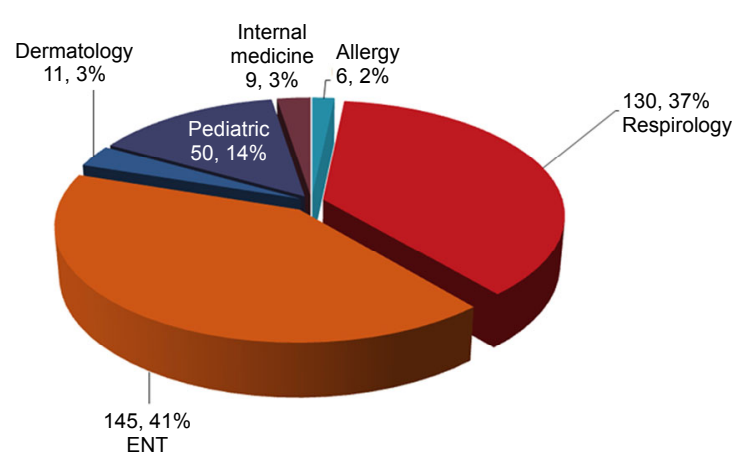

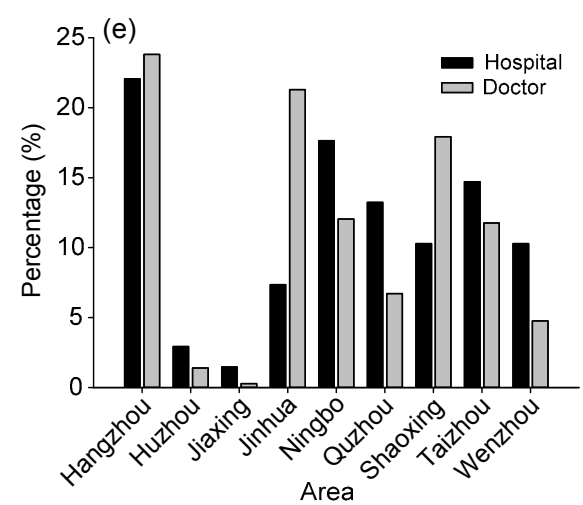

Fig. 1 General characteristics and distribution of doctors working in the diagnosis and treatment of allergic diseases (a) The age distribution of doctors, which reveals that the majority of them were at thirties and forties, and younger (less than 30 years) and older (more than 50 years) doctors only took a small portion; (b) The degree level distribution of doctors; (c) The distribution of doctors in hospitals at different levels; (d) The specialty distribution of doctors; (e) The distribution of doctors and hospitals from nine geographic areas

majority of doctors working in allergic diseases were in their forties, and the percentages of doctors in their twenties and thirties were at a similar level, which was about $20 \%$ (Fig. 2b). This data is consistent with the data of doctors' work-time. As shown in Fig. 2c, the majority of doctors in Respirology and ENT specialties had worked for 10 to 20 years with a percentage of $32 \%$ and $49 \%$, followed by doctors who had worked for 20 to 30 years at $26 \%$ and $21 \%$, respectively. The percentages of less experienced doctors with a work-time of less than 5 years and 5 to 10 years were similar at about $20 \%$ in the Respiratory specialty, but in the specialty of ENT, the percentage of doctors with a work-time less than 5 years only was about $12 \%$, clearly lower than that of doctors who had worked for 5 to 10 years. The doctors with the specialty of Pediatrics demonstrated a different pattern with a peak work-time of 20 to 30 years, higher than those with a work-time of less than 5 years and 5 to 10 years. Nevertheless, the majority of doctors were attending doctors (40\%-60\%) (Fig. 2d).
Fellows were more prevalent than residents in the Respirology and ENT specialties, however, less than residents in the Pediatrics specialty.

\subsection{Performance of diagnostic methods in hospi- tals at different levels and from different areas}

In general, about $60 \%$ of the hospitals had performed ssIgE assays, while the application of ImmunoCAP was much lower, only about $22 \%$ (Fig. 3). Nearly half of the hospitals had applied SPT in allergy diagnosis.

The performance status of these diagnostic methods varied in hospitals at different levels or from different areas. Despite the highest proportion of all these methods in municipal hospitals (supplemental material Fig. S1), SPT was performed primarily in district hospitals, followed by municipal and provincial hospitals almost at an equal level of $50 \%$, while only $9 \%$ of county hospitals applied this examination (Fig. 4a). As for the ssIgE assay, $100 \%$ of provincial hospitals provided this service, and more than $60 \%$ of 

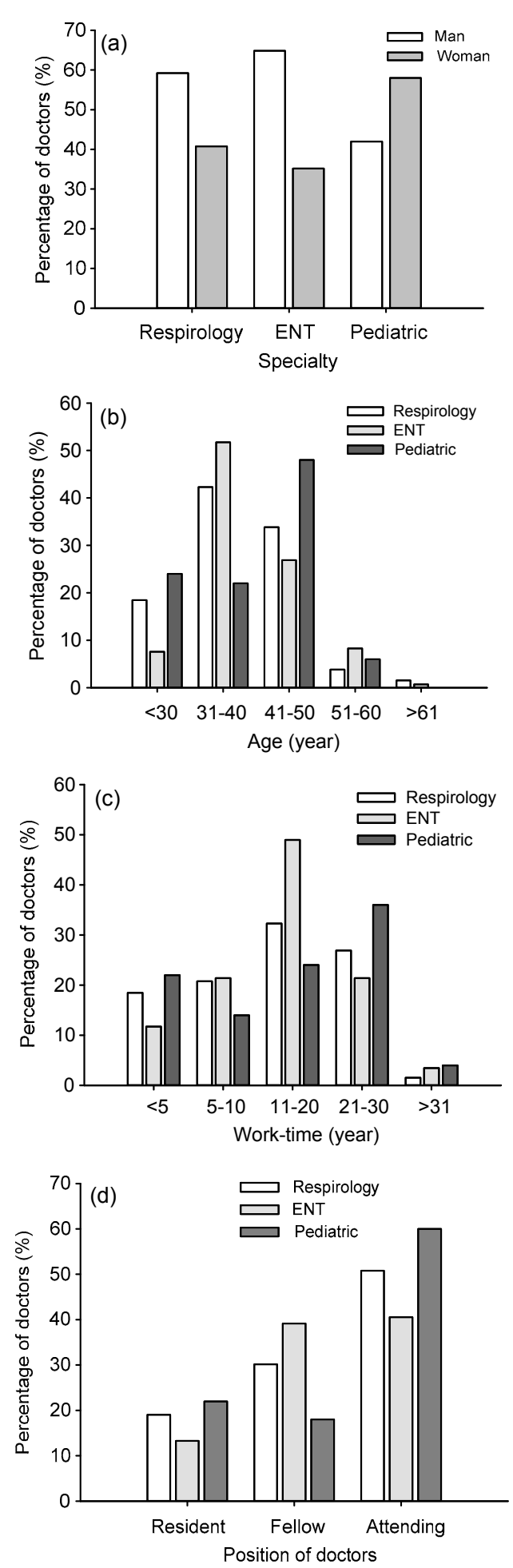

Fig. 2 Characteristics of doctors working in allergic diseases in the specialties of Respirology, ENT, and Pediatrics

The gender distribution (a), age distribution (b), work-time (c), and the position (d) of doctors from the specialties of Respirology, ENT, and Pediatrics

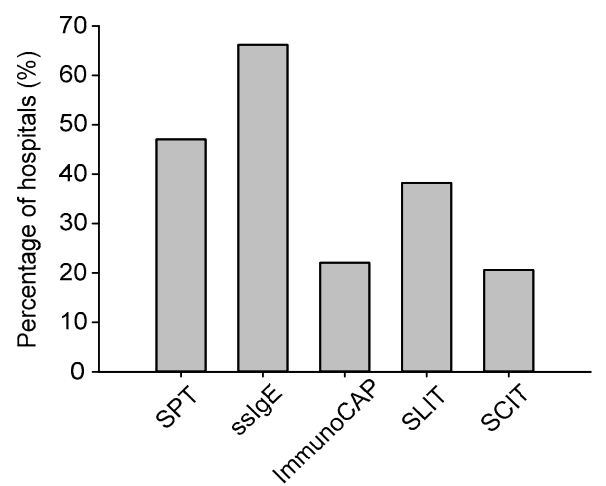

Fig. 3 Carryout of allergen measurements and allergen specific immunotherapy

The percentage of hospitals that performed ssIgE assay was much higher than that of hospitals that performed SPT. However, only $22 \%$ of hospitals had carried out ImmunoCAP. The percentage of hospitals that prescribed SLIT was higher than that of hospitals that prescribed SCIT

county and municipal hospitals also provided this service. Even in district hospitals, one third had provided it (Fig. 4b). However, the application of ImmunoCAP was not so widely provided. Despite $45 \%$ of the county hospitals using this method, only $21 \%$ of municipal hospitals and $11 \%$ of provincial hospitals had performed it. None of the district hospitals had this assay (Fig. 4c). More than half of the provincial hospitals and one third of the district and municipal hospitals performed both the SPT and ssIgE methods. However, only $9 \%$ of the county hospitals performed both (Fig. 4d). SPT was performed in all hospitals from Wenzhou and Jiaxing areas, while this practice was not started yet in the Huzhou, Jinhua, or Taizhou area. It was performed in $66 \%$ of hospitals from Hangzhou and Ningbo, followed by $44 \%$ of hospitals from Shaoxing and $22 \%$ of hospitals from Quzhou (Fig. 5a). Measurement of ssIgE was performed in $100 \%$ of hospitals from Jiaxing, Jinhua, and Taizhou areas, followed by $86 \%$ from Hangzhou, 57\% from Shaoxing and Wenzhou, and 50\% from Huzhou and Ningbo. And less than $10 \%$ of the hospitals from Quzhou had carried out this practice (Fig. 5b). Hospitals from the areas of Jiaxing, Jinhua, Ningbo, Quzhou, and Shaoxing had not carried out ImmunoCAP yet. And the application of this method in Hangzhou was surprisingly low with the application being provided in only $13 \%$ of the hospitals. On the contrary, all of the hospitals from Taizhou, $50 \%$ of the hospitals from Huzhou and $28 \%$ of the hospitals from Wenzhou had applied it (Fig. 5c). 

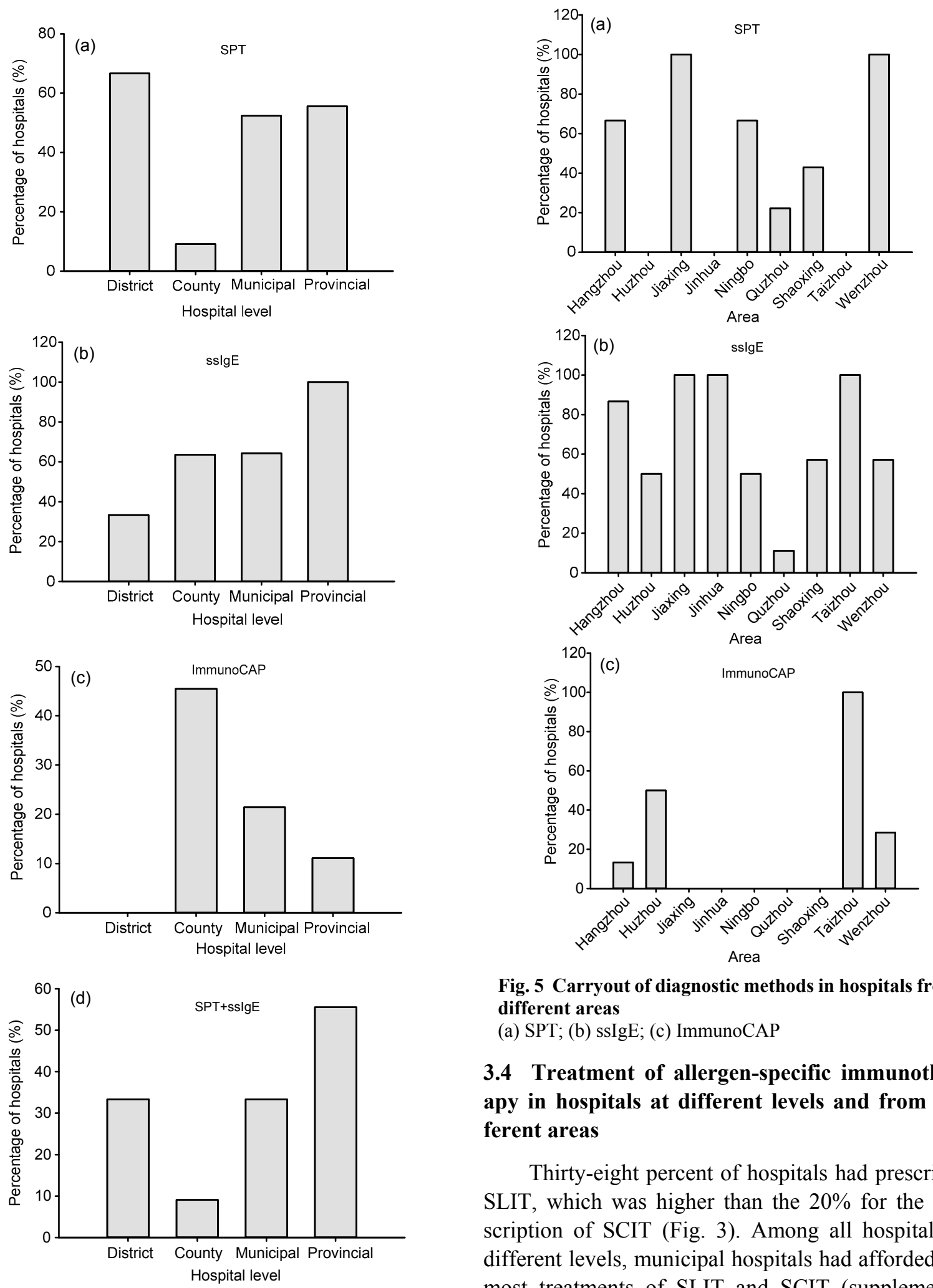

Fig. 5 Carryout of diagnostic methods in hospitals from different areas

(a) SPT; (b) ssIgE; (c) ImmunoCAP

\subsection{Treatment of allergen-specific immunother- apy in hospitals at different levels and from dif- ferent areas}

Thirty-eight percent of hospitals had prescribed SLIT, which was higher than the $20 \%$ for the prescription of SCIT (Fig. 3). Among all hospitals at different levels, municipal hospitals had afforded the most treatments of SLIT and SCIT (supplemental material Fig. S2). Fifty percent of municipal hospitals

Fig. 4 Carryout of diagnostic methods in hospitals at different levels and one third of district hospitals had carried out SLIT, (a) SPT; (b) ssIgE; (c) ImmunoCAP; (d) SPT+ssIgE while it was performed in only $22 \%$ of provincial 

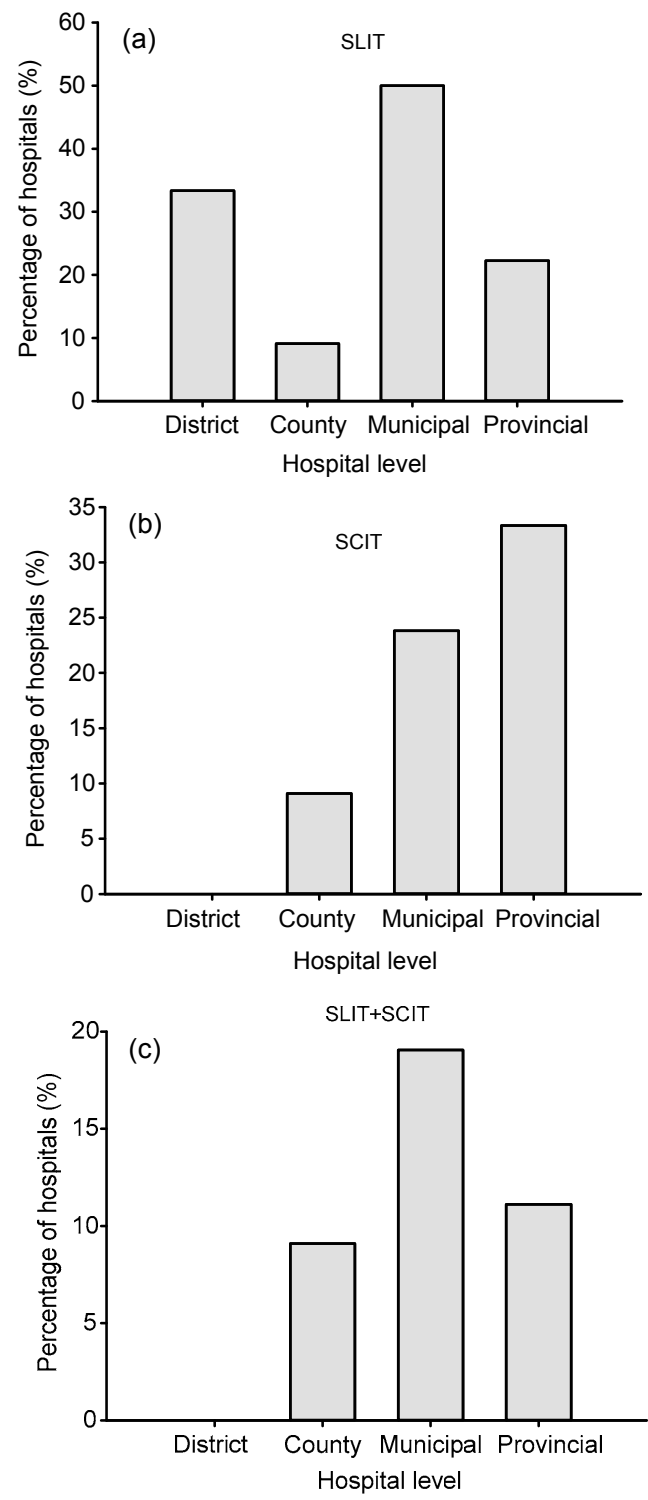

Fig. 6 Carryout of treatments in hospitals at different levels

(a) SLIT; (b) SCIT; (c) SLIT+SCIT

hospitals and $9 \%$ of county hospitals (Fig. 6a). The performance of SCIT was much less. One third of the provincial hospitals and $23 \%$ of the municipal hospitals had carried out SCIT, followed by $9 \%$ of county hospitals. No district hospitals had developed SCIT (Fig. 6b). As for the performance of both treatments, municipal hospitals had the highest ratio with $19 \%$, followed by provincial hospitals $(11 \%)$ and county hospitals $(9 \%)$ (Fig. 6c). The comparison of data from different areas revealed that SLIT had been prescribed in $100 \%$ of hospitals from Wenzhou, $47 \%$ of hospitals from Hangzhou, about $40 \%$ of hospitals
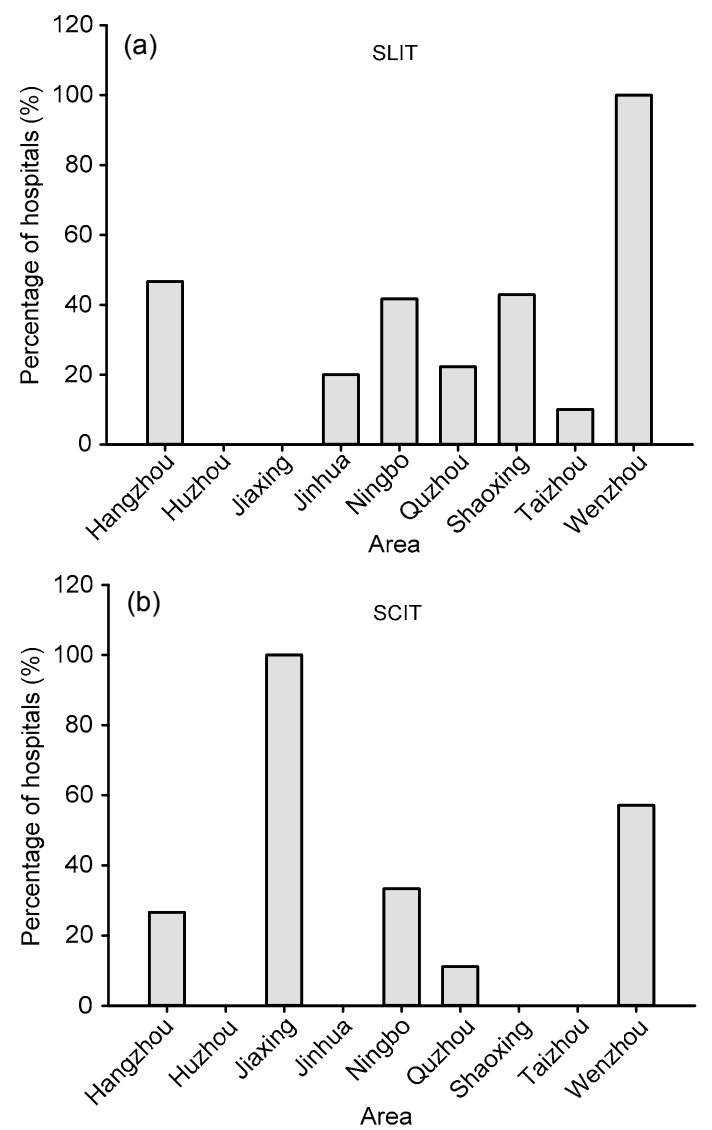

Fig. 7 Carryout of treatments in hospitals from different areas

(a) SLIT; (b) SCIT

from Ningbo and Shaoxing, and about $20 \%$ of hospitals from Jinhua and Quzhou areas (Fig. 7a). The prescription of SCIT was highest in Jiaxing area with $100 \%$ of the hospitals, followed by Wenzhou $(57 \%$ of the hospitals), Ningbo (33\% of the hospitals), and Hangzhou (26\% of the hospitals). Quzhou was at a very low level with only $11 \%$ of hospitals having carried out SCIT (Fig. 7b).

\section{Discussion}

The current study surveyed the status of the diagnosis and treatments of allergic diseases in Zhejiang Province, including the doctor resources from different specialties and the performance conditions of diagnostic methods and allergen-specific immunotherapy. Furthermore, we also analyzed the variation in hospitals at different levels and from different areas. 
In general, the development of the allergy specialty is still in the very initial stages in Zhejiang Province. The limited number of doctor resources working in allergic diseases is the major obstacle. Only those who have experience and professional knowledge could develop the subspecialty through improving their clinical skills, introduction of new techniques, continuous education and knowledge delivery for the younger doctors, and so on. This task seems to us not an easy one to accomplish for the small group of only 357 doctors treating allergic diseases as we defined, with only one Allergy Department established so far. And there is also an unavoidable flaw in the infrastructure of the doctor resources. The main stream of doctors treating allergic diseases is in their thirties with only academic Bachelor's degrees. Most of them do not have any basic research experience in immunology and actually are lacking any clinical experience, both of which are essential for disease diagnosis and treatment (Schatz et al., 2006). Thus, the medical services provided for patients with allergic diseases are also prone to be limited to the local systems without comprehensive considerations by doctors from different specialties. This condition actually is very dangerous, especially for patients with food allergies or hymenoptera venom allergies. These patients usually go to the Department of Dermatology or Gastroenterology depending on what symptoms are predominant, and are often prescribed some medicine for symptom relief without further accurate diagnosis of allergens. Thus future potential anaphylaxis is hard to avoid. Doctors from the specialties of ENT, Respirology, and Pediatrics constituted the majority of doctor resources, especially the specialty of ENT that has the largest proportion. Allergic rhinitis and asthma are among the most prevalent allergic diseases and represent a big burden to society (Braman, 2006; Ozdoganoglu and Songu, 2012). The related research about allergen-induced respiratory symptoms and molecular mechanisms of allergic reactions have been conducted extensively, which has further led to the clinical practice of allergen-specific immunotherapy for respiratory allergies worldwide (Hurwitz, 1953). The worldwide delivery of guidelines and investigations via those organizations such as Allergic Rhinitis and its Impact on Asthma (ARIA), Global Initiative for Asthma (GINA), and GA ${ }^{2} L E N$ has dramatically improved the recognition of allergies by doctors of related specialties (Bousquet et al., 2008; Kroegel, 2009; Newson et al., 2014). By contrast, although many dermatologic diseases are considered allergic, few dermatologists treat patients while considering allergen detection and avoidance. Another reason is associated to the history of the development of allergy subspecialties in China. The initiation of the subspecialty of allergies in China was accomplished by Dr. Ye (2001) from the ENT Department, starting from the study of hay fever, and was marked as the foundation of the independent Allergy Department separated from the ENT Department in Beijing Union Hospital fifty years ago. Therefore, doctors from the ENT specialty first led the work for allergies and were followed by those from Respirology. However, both specialties showed a shortage of young doctors to continue the work when looking into the composition of doctors. As a matter of fact, the development of allergies might be better in Pediatrics in the future. Doctors from Pediatrics usually treat both allergic rhinitis and asthma in children simultaneously, which means they would have more integrative knowledge about allergies in different organs. And the infrastructure for doctors working in allergic diseases is more reasonable than that from the other two specialties, with a more even proportion of younger doctors and experienced doctors. The knowledge from these experienced doctors is very important for younger doctors' improvement, especially for a new subspecialty without a strong systemic academic support.

The performance of diagnostic methods is quite underdeveloped with a surprising high ratio of ssIgE assays above SPT, but a poor application of ImmunoCAP. SPT is an in vivo test to assay the immediate IgE response to specific allergens, which reflects the function of mast cells that react to allergens ( $\mathrm{Li}, 2002)$. It is cheap and convenient, easy to operate, and is stated to be the preferred technique for IgE-mediated hypersensitivity by the Practice Parameter from 2008 (Bernstein et al., 2008), though training is needed for those who gather and interpret the results (Antunes et al., 2009; Bousquet et al., 2012). As an in vitro test, the ssIgE test is safer, without the influence of drugs and personal subjective bias (Siles and Hsieh, 2011). The quantification of ssIgE is also a useful tool for prediction of allergic 
symptoms (Sampson, 2001). Since the results are not interchangeable, it is necessary to try both for the hospitals of higher ranks. Otherwise, one method is enough for the diagnosis of allergen sensitization. Usually SPT is suitable for all hospitals including primary healthcare centers such as district and county hospitals, and ssIgE is more suitable for hospitals of higher ranks with enough capital and experienced technicians. Thus, SPT is expected to be applied more extensively and be popular than ssIgE and to be carried out in most of the hospitals. The main reason for the current opposite result is the economic factor. Although the operation of SPT needs trained nurses and a room equipped with rescue devices for potential allergic reactions even though it is very rare (Liccardi et al., 2006), the charge for SPT is relatively low. The assay of ssIgE takes less labor and space with a machine that completes almost all of the whole processes, and this assay approach incurs much more in charges. Therefore, ssIgE is preferred in most hospitals. And most of the ssIgE assays via the method of enzymelinked immunosorbent assay (ELISA) have very low cost. So it is easy to understand why the method of ImmunoCAP, one of the most popular ssIgE assay methods for its steady and reliable results (Bousquet et al., 1990), was not as popular as expected. The recognition for allergen detection methods is certainly another reason.

The prescription of allergen-specific immunotherapy is also not popular, with a higher proportion of SLIT above SCIT. Both SLIT and SCIT are effective immunoregulatory therapies for IgE-mediated diseases with long-term immunologic and clinical tolerance (Bauer and Rank, 2014; Roche and Wise, 2014). SCIT is a conventional treatment, which has more than 100 years of history with proved efficacy (Ring and Gutermuth, 2011). SLIT is a relatively new treatment that started in 1986, however with comparable immunologic mechanisms (Scadding et al., 2010; James et al., 2011). The potential side effects of anaphylaxis in SCIT largely restricted its clinical application, mainly because of the concerns about its safety raised by patients as well as doctors. And the treatment of SCIT should be performed at hospitals with well-trained nurses and doctors, as well as enough space and complete equipment in the case of a need for an anaphylaxis rescue, even if the probability is less than one of two million or two million eight hundred thousand (Reid et al., 1993). That could explain why provincial hospitals have the highest percentage of SCIT, and no district hospitals prescribe it. In contrast, SLIT is much more convenient and safer for patients, allowing for self-administration at home (Bauer and Rank, 2014). So it is applied more extensively and prescribed in even district hospitals.

The development of the allergy subspecialty is varied in hospitals at different levels and from different areas. Municipal hospitals are the main stream for allergic disease diagnosis and treatment, with the highest number of doctors and wider application of diagnostic methods and treatment. One reason is that the absolute number of municipal hospitals is definitely higher than that of provincial hospitals, and its technique and doctor resources are much stronger than those of county and district hospitals. Another reason is the shortage of allergists/immunologists in provincial hospitals. Thus, provincial hospitals did not play a leading role in allergy specialization. However, the news is that the concept of allergies has already been acknowledged by various parts of primary healthcare centers, albeit weak, which means that further continuous education and training for doctors in these hospitals is becoming realistic. Improving allergy management in these primary healthcare centers is very important as Jutel et al. (2013) stated, considering the huge population of patients at these primary healthcare centers.

The data for the prevalence of allergic diseases in Zhejiang Province is very limited, but surely it is not the main reason that caused the different developmental levels of allergy subspecialties. The whole level of medicine and economic status should be the major cause. Hangzhou and Wenzhou play the leading roles with the strongest doctor resources providing comprehensive medical service for diagnostic methods and treatments, mainly due to their geographic advantages with the location of most provincial hospitals and/or medical schools. Jiaxing and Huzhou areas, which are just adjacent to Hangzhou in geography and presumed to have similar prevalence and profiles of allergic diseases, by contrast, fall far behind other areas with the least number of hospitals, doctor resources, diagnostic methods, and allergenspecific immunotherapy. Other areas such as Ningbo area, where the allergy subspecialty is well developed, also have a close liaison with medical schools, which 
is also important for the practice of allergy subspecialties (van Metre, 1979). Although the initial origination of allergy studies is first from practice, the further development still needs the complete system of theory (International Association of Allergology, 1977). Medical schools are a good base for the study and delivery of this knowledge. So more efforts should be paid to the continuous education of allergy studies in these areas in the future.

\section{Conclusions}

In summary, our survey revealed that the development of allergy specialties in Zhejiang Province is in its very initial stages. The specialties of ENT, Respirology, and Pediatrics are the main stream to provide the medical service for diagnosis and treatment of allergic diseases, and the specialty of Pediatrics has better infrastructure of doctors for further development. The development of allergy subspecialties varied in hospitals at different levels and from different areas, the continuous education of allergies could be extended to primary healthcare centers and more efforts should be paid to those areas with poor medical resources.

\section{Acknowledgements}

We sincerely thank Dr. Xin-guo JIANG (Department of Medicine, VA Palo Alto Health Care System/Stanford University School of Medicine, USA) for providing valuable comments and language revisions for this study.

\section{Compliance with ethics guidelines}

Hui-ying WANG, Yi-hong SHEN, Xu-yan YANG, Lan-fang TANG, and Jian-ying ZHOU declare that they have no conflict of interest.

This article does not contain any studies with human or animal subjects performed by any of the authors.

\section{References}

Antunes, J., Borrego, L., Romeira, A., et al., 2009. Skin prick tests and allergy diagnosis. Allergol. Immunopathol., 37(3):155-164. [doi:10.1016/S0301-0546(09)71728-8]

Asher, M.I., Montefort, S., Bjorksten, B., et al., 2006. Worldwide time trends in the prevalence of symptoms of asthma, allergic rhinoconjunctivitis, and eczema in childhood: ISAAC Phases One and Three repeat multicountry cross-sectional surveys. Lancet, 368(9537): 733-743. [doi:10.1016/S0140-6736(06)69283-0]

Bauer, C.S., Rank, M.A., 2014. Comparative efficacy and safety of subcutaneous versus sublingual immunotherapy.
J. Allergy Clin. Immunol., 134(3):765-765.e2. [doi:10. 1016/j.jaci.2014.07.024]

Bergmann, K.C., 2014. Milestones in the 20th century. In: Bergmann, K.C., Ring, J. (Eds.), History of Allergy. Chem Immunol Allergy. Basel, Karger, Vol. 100, p.27-45. [doi:10.1159/000358478]

Bernstein, I.L., Li, J.T., Bernstein, D.I., et al., 2008. Allergy diagnostic testing: an updated practice parameter. Ann. Allergy Asthma Immunol., 100(3 Suppl. 3):S1-S148. [doi:10.1016/S1081-1206(10)60305-5]

Bousquet, J., Chanez, P., Chanal, I., et al., 1990. Comparison between RAST and Pharmacia CAP system: a new automated specific IgE assay. J. Allergy Clin. Immunol., 85(6):1039-1043. [doi:10.1016/0091-6749(90)90048-9]

Bousquet, J., Khaltaev, N., Cruz, A.A., et al., 2008. Allergic rhinitis and its impact on asthma (ARIA) 2008. Allergy, 63(S86):8-160. [doi:10.1111/j.1398-9995.2007.01620.x]

Bousquet, J., Heinzerling, L., Bachert, C., et al., 2012. Practical guide to skin prick tests in allergy to aeroallergens. Allergy, 67(1):18-24. [doi:10.1111/j.1398-9995.2011.027 28.x]

Braman, S.S., 2006. The global burden of asthma. Chest, 130(1_Suppl.):4S-12S. [doi:10.1378/chest.130.1_suppl.4S]

Burks, A.W., Calderon, M.A., Casale, T., et al., 2013. Update on allergy immunotherapy: American Academy of Allergy, Asthma \& Immunology/European Academy of Allergy and Clinical Immunology/PRACTALL consensus report. J. Allergy Clin. Immunol., 131(5):1288-1296.e3. [doi:10. 1016/j.jaci.2013.01.049]

Cox, L., Esch, R.E., Corbett, M., et al., 2011. Allergen immunotherapy practice in the United States: guidelines, measures, and outcomes. Ann. Allergy Asthma Immunol., 107(4):289-299. [doi:10.1016/j.anai.2011.06.018]

Fornadley, J.A., 2014. Skin testing for inhalant allergy. Int. Forum Allergy Rhinol., 4(Suppl. 2):S41-S45. [doi:10. 1002/alr.21393]

Hurwitz, S.H., 1953. The development of specialization in allergy: a historical review and a view ahead. Calif. Med., 78(3):216-221.

International Association of Allergology, 1977. Training and specialization in allergology (clinical allergy). Clin. Allergy, 7(3):205-209. [doi:10.1111/j.1365-2222.1977. tb01444.x]

James, L.K., Shamji, M.H., Walker, S.M., et al., 2011. Long-term tolerance after allergen immunotherapy is accompanied by selective persistence of blocking antibodies. J. Allergy Clin. Immunol., 127(2):509-516.e5. [doi:10.1016/j.jaci.2010.12.1080]

Jutel, M., Angier, L., Palkonen, S., et al., 2013. Improving allergy management in the primary care network-a holistic approach. Allergy, 68(11):1362-1369. [doi:10. 1111/all.12258]

Kroegel, C., 2009. Global Initiative for Asthma (GINA) guidelines: 15 years of application. Expert Rev. Clin. Immunol., 5(3):239-249. [doi:10.1586/eci.09.1]

Krouse, J.H., Mabry, R.L., 2003. Skin testing for inhalant allergy 2003: current strategies. Otolaryngol. Head Neck Surg., 129(4):S33-S49. [doi:10.1016/S0194-5998(03)013 98-6] 
Li, J., Sun, B., Huang, Y., et al., 2009. A multicentre study assessing the prevalence of sensitizations in patients with asthma and/or rhinitis in China. Allergy, 64(7):10831092. [doi:10.1111/j.1398-9995.2009.01967.x]

Li, J.T., 2002. Allergy testing. Am. Fam. Physician, 66(4): 621-624.

Liccardi, G., D'Amato, G., Canonica, G.W., et al., 2006. Systemic reactions from skin testing: literature review. $J$. Investig. Allergol. Clin. Immunol., 16(2):75-78.

Marshall, G.D., 2007. The status of US allergy/immunology physicians in the 21st century: a report from the American Academy of Allergy, Asthma \& Immunology Workforce Committee. J. Allergy Clin. Immunol., 119(4):802-807. [doi:10.1016/j.jaci.2007.01.040]

Newson, R.B., van Ree, R., Forsberg, B., et al., 2014. Geographical variation in the prevalence of sensitization to common aeroallergens in adults: the GA ${ }^{2} \mathrm{LEN}$ survey. Allergy, 69(5):643-651. [doi:10.1111/all.12397]

Ozdoganoglu, T., Songu, M., 2012. The burden of allergic rhinitis and asthma. Ther. Adv. Respir. Dis., 6(1):11-23. [doi:10.1177/1753465811431975]

Reid, M.J., Lockey, R.F., Turkeltaub, P.C., et al., 1993. Survey of fatalities from skin testing and immunotherapy 1985-1989. J. Allergy Clin. Immunol., 92(1):6-15. [doi:10. 1016/0091-6749(93)90030-J]

Ring, J., Gutermuth, J., 2011. 100 years of hyposensitization: history of allergen-specific immunotherapy (ASIT). Allergy, 66(6):713-724. [doi:10.1111/j.1398-9995.2010. 02541.x]

Roche, A.M., Wise, S.K., 2014. Subcutaneous immunotherapy. Int. Forum Allergy Rhinol., 4(Suppl. 2):S51-S54. [doi:10. 1002/alr.21382]

Sampson, H.A., 2001. Utility of food-specific IgE concentrations in predicting symptomatic food allergy. J. Allergy Clin. Immunol., 107(5):891-896. [doi:10.1067/mai.2001.114708]

Scadding, G.W., Shamji, M.H., Jacobson, M.R., et al., 2010. Sublingual grass pollen immunotherapy is associated with increases in sublingual Foxp3-expressing cells and elevated allergen-specific immunoglobulin G4, immunoglobulin A and serum inhibitory activity for immunoglobulin Efacilitated allergen binding to B cells. Clin. Exp. Allergy, 40(4):598-606. [doi:10.1111/j.1365-2222.2010.03462.x]

Schatz, M., Leung, D.Y.M., Goldstein, S., 2006. Consultation and referral guidelines citing the evidence: how the allergist-immunologist can help. J. Allergy Clin. Immunol., 117(2 Suppl. 3):S495-S523. [doi:10.1016/j.jaci.2005.10. 047]

Siles, R.I., Hsieh, F.H., 2011. Allergy blood testing: a practical guide for clinicians. Cleve. Clin. J. Med., 78(9):585-592. [doi:10.3949/ccjm.78a.11023]

van Metre, T.E.Jr., 1979. Presidential address. The advancement of the knowledge and practice of allergy. J. Allergy Clin. Immunol., 64(4):235-241. [doi:10.1016/0091-6749(79)90 138-6]

Warner, J.O., Kaliner, M.A., Crisci, C.D., et al., 2006. Allergy practice worldwide: a report by the World Allergy Organization Specialty and Training Council. Int. Arch. Allergy Immunol., 139(2):166-174. [doi:10.1159/0000 90502]
Wen, L.P., 2013. Summary of 2013 annual congress of Chinese Allergy Society. Chin. J. Allergy Clin. Immunol., 8(1):83-85 (in Chinese). [doi:10.3969/j.issn.1673-8705. 2014.01.017]

Ye, S.T., 2001. The start and development of allergy specialty in China. Chin. J. Microbiol. Immunol., 21(Suppl.):1-3 (in Chinese).

Yin, J., 2014. A new turning point for the development of allergy specialty in China. Chin. J. Allergy Clin. Immunol., 8(1):1-2 (in Chinese). [doi:10.3969/j.issn.1673-8705.2014. 01.001]

Zhejiang Provincial Bureau of Statistics, 2014. 2013 annual communique of the statistics of Zhejiang provincial economy and society development. Stat. Theory Pract., 3:4-10 (in Chinese). [doi:10.3969/j.issn.1674-8905.2014. 03.001]

Zuberbier, T., Lotvall, J., Simoens, S., et al., 2014. Economic burden of inadequate management of allergic diseases in the European Union: a GA ${ }^{2} \mathrm{LEN}$ review. Allergy, 69(10): 1275-1279. [doi:10.1111/all.12470]

\section{List of electronic supplementary materials}

Fig. S1 Proportion of hospitals at different levels carrying out the diagnostic methods of SPT (a), ssIgE (b), ImmunoCAP (c), and SPT + ssIgE (d)

Fig. S2 Proportion of hospitals at different levels carrying out the treatments of SLIT (a), SCIT (b), and SLIT+SCIT (c)

\section{中文概要}

题 目: 浙江省过敏性疾病的诊断和治疗现状的横断面 研究

目 的: 通过问卷调查浙江省过敏性疾病的诊断和治疗状 况, 包括治疗疾病的医生资源、诊断手段和特异 性免疫治疗的开展情况, 为将来过敏学科的发展 提供真实可靠的信息。

创新点: 国内首次通过问卷调查, 明确浙江省过敏性疾病 的诊治现状, 以期为未来发展学科的政策提供信 息。

方 法: 设计调查问卷, 定义诊治过敏性疾病的医生。问 卷按照不同的行政地区划分，分发到不同级别的 医院, 收集诊治过敏性疾病的医生和医院的全面 信息，然后进行分析。

结 论: 调查显示浙江省的过敏学科的发展还处于起始阶 段, 且存在地区间的发展不平衡。诊治过敏性疾 病的医生主要来自五官科、呼吸科和小儿科，过 敏原的诊断和特异性免疫治疗主要集中在省市 级医院。今后过敏学科的继续教育可扩展至初级 医疗保健机构, 并对缺乏医学资源的地区加大资 源投入。

关键词: 过敏性疾病; 诊断; 治疗; 过敏原特异性免疫治疗 